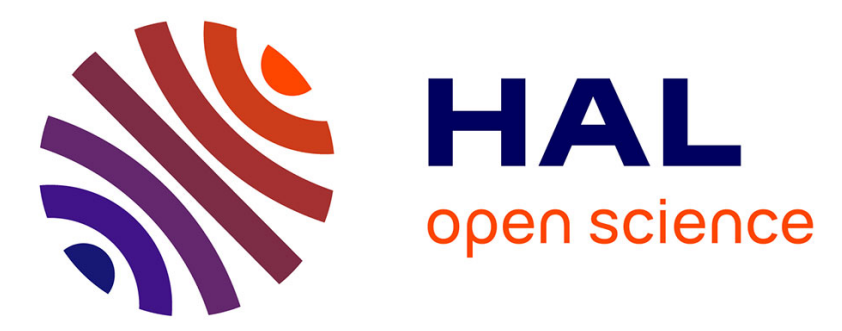

\title{
Characterizing and measuring path diversity of internet topologies
}

\author{
Renata Teixeira, Keith Marzullo, Stefan Savage, Geoffrey M. Voelker
}

\section{To cite this version:}

Renata Teixeira, Keith Marzullo, Stefan Savage, Geoffrey M. Voelker. Characterizing and measuring path diversity of internet topologies. ACM SIGMETRICS, Jun 2003, San Diego, United States. Proc. of ACM SIGMETRICS, 2003, 10.1145/781064.781069 . hal-01097527

\section{HAL Id: hal-01097527 \\ https://inria.hal.science/hal-01097527}

Submitted on 24 Dec 2014

HAL is a multi-disciplinary open access archive for the deposit and dissemination of scientific research documents, whether they are published or not. The documents may come from teaching and research institutions in France or abroad, or from public or private research centers.
L'archive ouverte pluridisciplinaire HAL, est destinée au dépôt et à la diffusion de documents scientifiques de niveau recherche, publiés ou non, émanant des établissements d'enseignement et de recherche français ou étrangers, des laboratoires publics ou privés. 


\title{
Characterizing and Measuring Path Diversity of Internet Topologies
}

\author{
Renata Teixeira*, Keith Marzullo, Stefan Savage, Geoffrey M. Voelker \\ Computer Science and Engineering, University of California, San Diego \\ \{teixeira,marzullo,savage,voelker\}@cs.ucsd.edu
}

\section{Categories and Subject Descriptors}

C.2.1 [Computer Communications and Networks]: Network Archtecture and Design — network topology

\section{General Terms}

Measurement

\section{INTRODUCTION}

The use of multiple network paths between a pair of hosts has been proposed for a wide variety of network technologies in order to achieve higher bandwidth in data transfers, to select paths with low latency, to balance load in the network, and to protect against failures.

Path diversity describes the number of disjoint paths between hosts and characteristics of those paths, such as path length, latency, and bottleneck bandwidth. Despite wide interest in such techniques, little work has been done that actually characterizes and measures the path diversity that exists in large-scale deployed packet networks like the Internet. Our work characterizes layer-3 path diversity ${ }^{1}$ in the Internet.

\section{PATH DIVERSITY IN THE INTERNET}

The Internet is an interconnection of autonomous systems. An autonomous system (AS) is a network under a single administrative entity, such as an Internet Service Provider (ISP), an enterprise, or a campus. Each ISP network is a collection of physical locations called Points of Presence, or PoPs. Customers connect to the ISP network in one of the ISP's PoPs. PoPs are interconnected by inter-PoP links. ASes interconnect at peering points. Routers that interconnect ASes exchange routing announcement updates using the Border Gateway Protocol (BGP), a policy-based protocol.

\footnotetext{
${ }^{*}$ Supported by Capes/Brazil

${ }^{1}$ Note that layer-3 path diversity is distinct from physicallevel path diversity: several virtual links may share a phys-
} ical link.
As a start, we characterize the path diversity between a pair of nodes in a network topology by the number of nodeand link-disjoint paths between them. The number of disjoint paths between two hosts in the Internet depends on where and how they connect to the network. Paths between two hosts in the same AS are determined by the AS's internal network infrastructure and by the intra-domain routing protocol. Paths between two hosts connected to different ASes are determined not only by the network infrastructure and routing policies inside each network on the path, but also by peering connections and inter-domain routing policies used by BGP. Thus, it is important to study path diversity both inside an ISP network and across multiple networks.

In our work, we analyze a set of network topologies (both individual AS and multiple-AS topologies). In this paper we present the results for two topologies: the topology of the Sprint network, which illustrates the path diversity inside an AS; and a topology derived from CAIDA, which illustrates the path diversity available across multiple ASes in the Internet.

\subsection{Path Diversity inside an ISP Network}

As an example, we study path diversity in the continental US Sprintlink topology. The exact topology was provided by Sprint under a non-disclosure agreement ${ }^{2}$. We study a PoPlevel topology that is composed of 17 PoPs. Each node in the topology represents the set of fully-meshed routers in a PoP. Links represent both active and backup IP links between PoPs, which allow us to assess the maximum potential for path diversity.

We measure path diversity for every pair of Sprintlink PoPs. Figure 1 shows the cumulative distribution function of the number of disjoint paths for all pairs of PoPs. The solid line represents the maximum possible number of paths for each pair of PoPs as determined by the minimum between the out-degree of the source PoP and the in-degree of the destination PoP.

Figure 1 shows that the exact Sprintlink topology has a high level of path diversity. Almost $90 \%$ of pairs of PoPs have at least four link-disjoint paths between them and $40 \%$ of pairs of PoPs are connected through eight or more linkdisjoint paths. For protection reasons, when there is more than one link between two PoPs, each of these links connects physically different routers. Hence, link-disjoint paths in this topology are also router disjoint.

\footnotetext{
${ }^{2} \mathrm{An}$ approximate topology can be found at http://www.sprint.net
} 


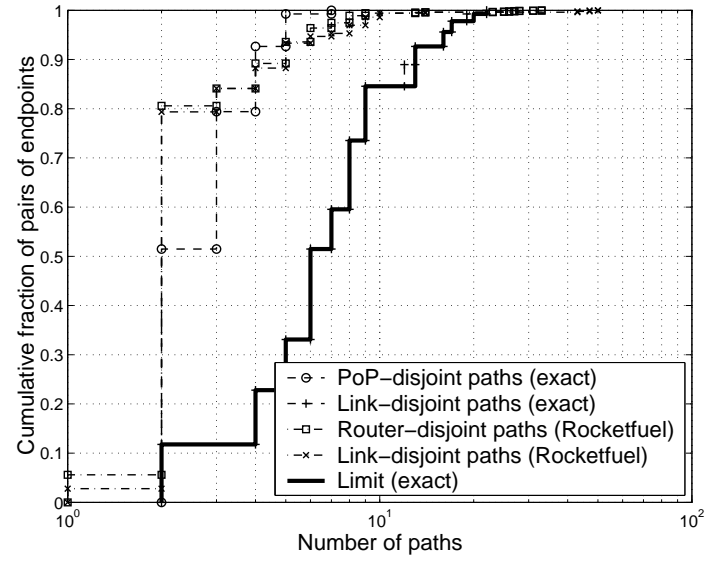

Figure 1: Path diversity in the Sprintlink topology.

Path diversity inside an ISP network depends on how ISPs engineer their network and thus it varies from one ISP to another. In an attempt to characterize path diversity for a larger set of ISPs, we also studied ISP topologies made publicly available by the Rocketfuel project [2]. These are router-level topologies extracted using end-to-end measurements. Since the topologies are generated by sampling, Rocketfuel infers the set of IP addresses belonging to a single router and only captures active links.

Overall, our results for the Rocketfuel topologies showed that there is significant variance of path diversity between different ISPs, but in general, tier-1 ISPs have at least two paths between an entry and an exit point in their network. Figure 1 also shows the results for the Rocketfuel-derived Sprint topology. Comparing the number of link-disjoint paths in the exact Sprint topology with the number of routerdisjoint paths measured by Rocketfuel for the same topology, we see that the path diversity in topologies measured by Rocketfuel is underestimated ${ }^{3}$. Rocketfuel gives us the paths that are simultaneously active between two PoPs, instead of the maximum set of available paths in the complete Sprint topology.

\subsection{Path Diversity across the Internet}

To analyze path diversity across multiple ASes, we use a topology from CAIDA[1]. CAIDA has been recording active measurements from various monitors scattered around the world since 1998. Paths in the CAIDA topology are composed of active links. In our study, we use a snapshot of a core of the CAIDA Internet topology collected from 16 monitors with over 57,000 nodes and nearly 200,000 edges.

We compute path diversity among the 16 monitors. Since most of the monitors have an out-degree of one, $90 \%$ of the pairs of monitors are connected by only one path. The lack of path diversity is not a property unique to CAIDA monitors: most hosts connected to the Internet are single-homed. This lack of diversity led us to investigate alternative definitions of path diversity. We define partially-disjoint paths as those for which there are some nodes that appear in more than one path. We identify the routers on the border of

\footnotetext{
${ }^{3}$ The fact that the number of disjoint paths in the Rocketfuel topology is larger than the maximum for some pair of nodes is a consequence of IP addresses that were not accurately mapped to the same router.
}

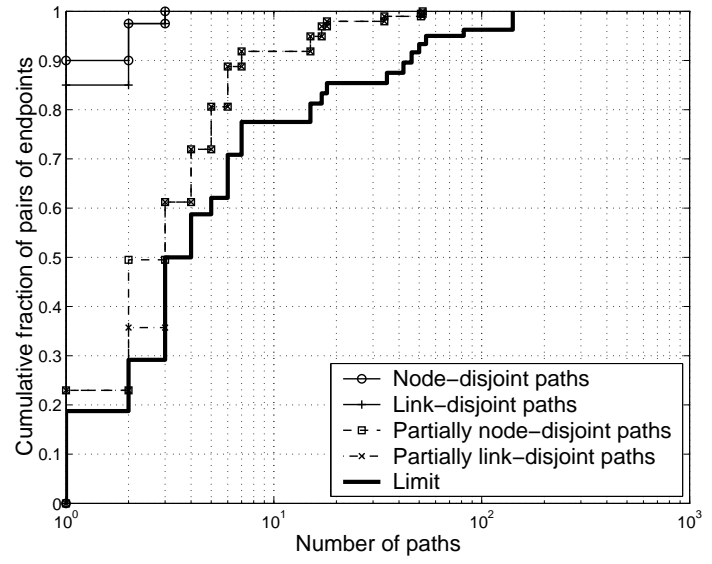

Figure 2: Cumulative distribution of the number of partially-disjoint paths in the CAIDA topology.

the ASes of the source and the destination, and compute path diversity for those nodes. This value represents the maximum path diversity available between the monitors' ASes.

Figure 2 presents the cumulative distribution of the number of partially-disjoint paths. It also shows the cumulative distribution of fully-disjoint paths and the limit imposed by the degree of the border nodes for comparison. The percentage of pairs of monitors connected by only one path drops to $24 \%$ when allowing nodes inside the ASes of the source and destination CAIDA monitors to be shared along the paths.

The CAIDA topology is constrained by routing policies and can only capture links that are active, therefore the path diversity presented in Figure 2 represents a lower bound of the number of disjoint paths for the CAIDA monitors. Path diversity in such a network is a useful approximation to the path diversity that an overlay network, for example, could exploit in practice if deployed on the Internet.

\section{CONCLUSION}

Our results show that there is potential for high level of path diversity inside an ISP, as illustrated by the Sprint network. The utilization of this path diversity, however, is limited by the routing protocols. Current intra-domain routing protocols use multiple paths in the event of a failure or for balancing load among equal-cost paths. Results from the CAIDA topology indicate that even though there is usually only one path between two end-hosts, there is significantly more path diversity across multiple ASes in the core of the Internet. This implies that in order to achieve higher endto-end path diversity, clients should be multi-homed.

This work is a first step toward a model of path diversity in the Internet. We are generalizing these results for a larger number of ISP topologies and inter-domain topologies collected from a varied set of monitors.

\section{REFERENCES}

[1] B. Huffaker, D. Plummer, D. Moore, and k claffy. Topology Discovery by Active Probing. In Symposium on Applications and the Internet (SAINT), Jan. 2002.

[2] N. Spring, R. Mahajan, and D. Wetherall. Measuring ISP Topologies with Rocketfuel. In Proceedings of $A C M$ SIGCOMM, Pittsburgh, PA, Aug. 2002. 